\title{
Lack of school requirements and clinician recommendations for human papillomavirus vaccination
}

\author{
Linda M. Niccolai, ${ }^{1}$ Anna L. North, ${ }^{1}$ Alison Footman, ${ }^{1}$ Caitlin E. Hansen ${ }^{2}$ \\ ${ }^{1}$ Department of Epidemiology of Microbial Diseases, Yale School of Public Health; ${ }^{2}$ Department of \\ Pediatrics, Yale School of Medicine, New Haven, CT, USA
}

Significance for public health

More than a decade after its introduction, human papillomavirus (HPV) vaccination coverage remains low in the United States despite a robust record of safety and effectiveness. A strong recommendation from a clinician is one of the most important determinants of uptake, yet clinicians often fail to provide effective recommendations. One potential barrier to effective recommendations by clinicians that has not been explicitly studied is the widespread lack of school entry requirements for HPV vaccination. In this qualitative study, we found the lack of requirements to be an important reason for clinicians' framing HPV vaccination recommendations as optional and non-urgent. Efforts are needed to strengthen the quality of clinicians' recommendations in a way that is focused on disease prevention and uncoupled from requirements. Additionally, greater support for HPV vaccination requirements among clinicians may be needed to successfully enact requirements in the future.

\section{Abstract}

Background: A strong recommendation from a clinician is one of the best predictors of human papillomavirus (HPV) vaccination among adolescents, yet many clinicians do not provide effective recommendations. The objective of this study was to understand how the lack of school entry requirements for HPV vaccination influences clinicians' recommendations.

Design and Methods: Semi-structured interviews with a purposive sample of 32 clinicians were conducted in 2015 in Connecticut USA. Data were analysed using an iterative thematic approach in 2016-2017.

Results: Many clinicians described presenting HPV vaccination as optional or non-urgent because it is not required for school entry. This was noted to be different from how other required vaccines were discussed. Even strong recommendations were often qualified by statements about the lack of requirements. Furthermore, lack of requirements was often raised initially by clinicians and not by parents. Many clinicians agreed that requirements would simplify the recommendation, but that parents may not agree with requirements. Personal opinions about school entry requirements were mixed.

Conclusions: The current lack of school entry requirements for HPV vaccination is an important influence on clinicians' recommendations that are often framed as optional or non-urgent. Efforts are needed to strengthen the quality of clinicians' recommendations in a way that remains strong and focused on disease prevention yet uncoupled from the lack of requirements that may encourage delays. Additionally, greater support for requirements among clinicians may be needed to successfully enact requirements in the future.

\section{Introduction}

Human papillomavirus (HPV) infection is an established risk factor for cancers of the anogenital tract and the oropharynx. ${ }^{1}$ Vaccines against HPV are safe, effective, and have demonstrated population-level impacts on reducing HPV infections and associated diseases in many countries, ${ }^{2,3}$ yet coverage remains low in the United States (US) more than a decade after their introduction. In 2016 , only $50 \%$ of girls and $38 \%$ of boys aged 13-17 years were up-to-date with the national Advisory Committee on Immunization Practices' (ACIP) recommended HPV vaccination series, far below the Healthy People 2020 goals of $80 \%{ }^{4}$ This is in contrast to coverage rates of $88 \%$ for tetanus/diphtheria/pertussis (Tdap) and $82 \%$ for meningococcal (MCV4) vaccines that share the same ACIP recommendation for routine administration at ages 11-12 years. ${ }^{4}$ Furthermore, HPV vaccination coverage is lower in the US than in many other developed countries: for example, countries in Northern Europe, Australia, and New Zealand have collectively achieved approximately $80 \%$ coverage with at least one dose among adolescent females. ${ }^{5}$

A key difference between the US and other countries that have achieved high coverage of HPV vaccination is their vaccine delivery systems. Countries such as Australia, Canada, and England have all achieved high coverage, generally $>80 \%$, through programs delivered in the context of universal health care systems and often through regional or national schoolbased programs that can reach a large number of children in a relatively short period of time. ${ }^{6-8}$ In contrast, the US has neither a national health care system nor a national school-based immunization program. Rather, immunizations are typically delivered in medical practices by primary care clinicians (practice-based delivery). This fragmented delivery system creates multiple possibilities for missed opportunities to immunization due to numerous challenges including varied practice-based policies and individual clinician beliefs and behaviours

In a practice-based vaccination delivery system such as that found in the US, a clinician's recommendation for vaccination is critically important. A strong recommendation from a clinician for HPV vaccination has been consistently reported as one of the most important facilitators of HPV vaccine uptake. ${ }^{9,10}$ However, research from the US has demonstrated that clinicians often present HPV vaccine as optional or with less urgency than other routinely recommended adolescent vaccines. ${ }^{11-15}$ Several reasons for this have been identified.

Clinicians may not provide effective recommendations because of their own lack of knowledge about vaccine safety or effectiveness, perceived lack of risk in their patients, and the fact that they rarely see HPV-associated cancers in their patients 
because of the long delay between infection and development of cancer. ${ }^{16,17}$ Clinicians may also be reluctant to discuss HPV vaccine with families because discussions may be perceived as uncomfortable if the sexually transmitted nature of HPV arises or clinicians may incorrectly assume that parents consider HPV vaccination to be unimportant. ${ }^{14,18}$ Clinicians may also anticipate parents' hesitancy due to parents' perceived lack of knowledge about the vaccine and safety concerns that could result in time-consuming conversations. ${ }^{14}$

Within the US, a notable difference among the three recommended vaccines for adolescents is the lack of widespread requirements for HPV vaccination for middle school entry. While ACIP recommendations are evidence-based, developed through expert review, and reflect public health guidance, they do not carry legal authority. On the other hand, state-level vaccination requirements for school entry are either administrative regulations or statutory laws, though exemptions for medical, religious, or philosophical reasons exist in most places. School entry requirements, sometimes referred to as mandates, are a widely used component of state vaccination policies for many childhood vaccines, and they have played an important role in both reducing the burden of diseases and in achieving more equitable coverage. ${ }^{19,20}$ Yet ten years after recommendations for routine vaccination for girls, only three jurisdictions out of 51 , all 50 states and District of Columbia, require HPV vaccination for entry to middle school including the District of Columbia, Rhode Island, and Virginia. ${ }^{21}$ In comparison, 50 jurisdictions currently require Tdap vaccination and 31 jurisdictions require MCV4 for middle school entry. Early efforts by some states to require HPV vaccination for school entry, which occurred soon after licensure and were considered politically and economically motivated, raised controversy. ${ }^{22}$ These efforts also occurred amidst substantial media coverage that fuelled debate about the about the importance of individual liberties and government control. It also raised concerns about requiring a vaccine for a sexually transmitted infection that was not likely to be transmitted in the school setting. Many of these sentiments have remained to present day reflected in on-going limited public support for school entry requirements. ${ }^{23,24}$

This qualitative study was conducted to understand clinicians' experiences with recommending HPV vaccination in the context of lack of school entry requirements. It also examined how clinicians anticipated future requirements might change their recommendations and/or acceptance by parents. Furthermore, because clinician support for requirements will be key to their enactment and successful implementation, their own personal opinions about school entry requirements were elicited.

\section{Design and Methods}

\section{Study sample}

Qualitative methods were chosen for this study to provide deeper insight into clinicians' thoughts and opinions that underlie their behaviours related to recommending HPV vaccination. This study was conducted in Connecticut, a state without a school entry requirement for HPV vaccination. Qualitative interviews were conducted in 2015 with clinicians who provide immunizations to adolescents. Clinicians were recruited through purposive sampling to ensure representation of different types of practices (private practice, hospital-based, and community clinics), specialties (pae- diatrics and family medicine), and different regions of the state (eight counties). This approach allowed us to capture a range of opinions across these characteristics and to ensure no prevailing theme would be missed. Clinicians were identified through professional networks and a state-wide database of active, practicing clinicians.

\section{Data collection}

A semi-structured interview guide was developed to elicit information about general practices and experiences regarding HPV vaccination. The guide also included a question and followup probes related to school entry requirements for HPV vaccination (Table 1). The guide was reviewed by two clinicianresearchers, pilot tested with two clinicians (not included in the sample), and subsequently revised. Interviews were conducted by phone, audio recorded, and transcribed.

\section{Data analysis}

Data analysis was conducted during 2016-2017 using an iterative thematic approach. ${ }^{25}$ This analytic approach was selected to identify key themes, or observed units of meaning, from the data (interview transcripts) through an integrated process of text segmentation, content coding, and theme identification. An initial coding guide was developed based on key topics in the interview guide and applied to segments of the transcripts by three investigators. Initial discrepancies in coding were resolved through discussion until consensus was achieved. Subsequently, all transcripts were coded by at least one member of the research team using Atlas.ti software (Version 7.5.4). Code reports were generated for topics related to school entry requirements, and the full research team then identified, discussed, and refined emergent themes. Results were examined by the clinicians' practice setting classified into three groups as follows: hospital-based clinic, federally qualified health centre, or $\geq 50 \%$ public insurance (subsequently referred to as public setting); $11-49 \%$ public insurance (mixed setting); and $\leq 10 \%$ public insurance (private setting). This practice setting characteristic was deemed important to consider because of known differences in HPV vaccination coverage by income. ${ }^{4}$ The study protocol was deemed exempt from review by the institutional review board for human subjects research at Yale University, and all ethical principles regarding autonomy, respect, informed consent, confidentiality, and data security were followed.

\section{Results}

A total of 32 clinicians completed interviews. A majority of the sample was female $(69 \%)$, and the median age was 55 years (range: 31 to 75 years). Most of the sample was physicians $(88 \%)$ with fewer nurse practitioners $(12 \%)$, and $88 \%$ were trained in paediatrics and $12 \%$ trained in family medicine. Three-quarters of the sample worked in private practice, and the remaining $25 \%$ worked in either hospital-based clinics or community health centres. The estimated percent of patients with public insurance was as follows: $31 \%$ reported $0-10 \%, 41 \%$ reported $11-49 \%$, and $28 \%$ reported $>50 \%$. Emergent themes are discussed below with illustrative quotes presented in Tables 2-4.

Many clinicians acknowledged that lack of school entry requirements led them to present HPV vaccination as recommended but not required. Some clinicians noted that because of the lack of a school entry requirement, they made a different type of recommendation for HPV vaccine than for Tdap or MCV4 vaccines. 
Noting that parents might not feel obliged to accept HPV vaccination, clinicians felt that the discussion around vaccination was different. One clinician remarked that HPV vaccination was offered as a choice. These views were expressed by providers across the range of practice settings.
Several clinicians described how they stated that HPV vaccination was not required for school in their initial presentation of the vaccine, and not in response to the issue being raised by parents. When asked the general question about how they usually introduced HPV vaccination to parents, clinicians described offering

Table 1. Sample questions from interview guide.

General questions about HPV vaccination

Questions specific to school entry requirements attendance
1. How do you usually offer HPV vaccine when you see an eligible patient?

2. Does your recommendation for HPV vaccine differ from your recommendation for other vaccines? Probe: If so, how?

3. In your opinion, what are the barriers to parents for vaccinating their children against HPV?

4. What do you think would help increase rates of HPV vaccination?

1. How would your experience offering HPV vaccine be different if HPV were mandated for school? Probe: Do you think a mandate would raise vaccination rates? Probe: In your opinion, do you think a mandate would be a good or bad policy move?

Table 2. Impact of lack of school entry requirement on recommendation for HPV vaccination.

Themes Illustrative quotes

Often results in a different type

of recommendation than for other vaccines

Initial discussion sometimes leads with statements about lack of requirement

Even strong recommendations are qualified by statements that it is not required

Few clinicians discussed the need for longer conversations
Well, it is [different], because I think the Menactra's required for school—for the middle school kids. It's required for entry to college... It's something that has to go on the school form. (P4) This [HPV vaccination] doesn't have that sense of obligation. The sell is a little different. $\mathrm{HPV}$ is the only one that is offered as a choice. (P14)

Well, I say these are required and this one's recommended. I mean, they could still go to seventh grade or sixth grade...It's not required. I tell them that. (P22)

Oh, I say, 'Well, we'd like to offer the HPV vaccine. It's a recommended but not required vaccine.' (P19) With their 11-year physical, we routinely introduce it. At the same time, we also have Tdap and Menactra that are required. We say we have the HPV, and we say what it prevents; in girls, genital warts, and in boys in both girls and boys, genital warts, and in girls, cervical cancer. We tell them that it's recommended. (P22)

I'll tell parents it's not required for school, which it's not, but that it's - there's a big health benefit, you know, it's a carcinogenic virus. (P1)

When you're telling parents that, "Well, this [other vaccines] has to be done because it's required by the state for school entry. The other one [HPV] isn't. It's an optional vaccine, but it is one we recommend." (P19) Just they're 11 now, there's three vaccines we need to talk about. Two the school is gonna require and one that we - isn't required by school but we still think it's a smart idea and we like to keep people healthy and this is what it is. It's very straightforward. (P31)

Because it is not a mandated vaccine, it often times takes parents a little longer to consent. 'Here's your Tdap, it's required for school entry.' It's usually a very short conversation... For HPV, because it's not a mandated vaccine, it tends to involve more of a discussion. (P2)

\section{Table 3. Anticipated impact of enacting a requirement on future recommendations.}

\begin{tabular}{|c|c|}
\hline Themes & Illustrative quotes \\
\hline $\begin{array}{l}\text { Majority reported that recommendation } \\
\text { would be simplified and conversation } \\
\text { would be shortened }\end{array}$ & $\begin{array}{l}\text { If we could start with 'Here's the HPV vaccine. It's required prior to ninth grade.', } \\
\text { it would be much easier for parents. Parents in general don't question it as much. (P12) } \\
\text { We can discuss what it's for but the bottom line is they have to have it, so that makes it a lot easier. (P17) } \\
\text { I mean, we'd still have to - we have to talk about it anyway... But if it was required for school, maybe the people } \\
\text { who need to be convinced wouldn't need to be convinced as much. We wouldn't have to spend as much } \\
\text { time trying to talk people into it. (P23) }\end{array}$ \\
\hline $\begin{array}{l}\text { Fewer reported conversation } \\
\text { could become more difficult }\end{array}$ & $\begin{array}{l}\text { I think if it were mandated, it's going to be, 'You're telling me to give my child a shot for a sexually transmitted } \\
\text { disease? Come on.' It's going to make people annoyed. (P1) } \\
\text { Yeah, I think the conversation would get longer. I think some of those families that don't wanna do it, } \\
\text { the conversation would go on and on. (P29) }\end{array}$ \\
\hline Few also reported no change & $\begin{array}{l}\text { Because I treat all vaccines the same regardless of state requirements. I do what's best for the patient. (P25) } \\
\text { The approach probably wouldn't be any different. I'd still wanna explain why we want to do it as opposed to well, } \\
\text { school says you have to so we're just gonna do 'em and that's it. (P31) }\end{array}$ \\
\hline $\begin{array}{l}\text { Requirement would increase parental } \\
\text { acceptance, even if they disagreed }\end{array}$ & $\begin{array}{l}\text { I think many of our parents are happy to do anything that they think they have to do. } \\
\text { Then they don't have to think about it. (P8) } \\
\text { I think our adoption rate would go up significantly although we may have some frustrated or you know, aggravated } \\
\text { parents who disagree with it. I do think it would help. (P9) } \\
\text { They [parents] wouldn't consider it optional any more, as optional as they seem to consider it now. (P10) } \\
\text { If HPV were a school requirement, I am pretty sure that people would go along with it. (P21) } \\
\text { I think it would [work]. I think you'd get a lot of grumpy people. (P27) } \\
\text { My own personal opinion, I think it sends them [parents] a strong message that this is important. (P28) }\end{array}$ \\
\hline
\end{tabular}


the HPV vaccine as one that was recommended but not required. In this way, it was often presented as an additional vaccine and one that was separated from Tdap and MCV4 vaccines at the outset of the conversation about the three immunizations that share the same recommendation for routine administration at the same age of 1112 years. Often, this conversation occurred in an unsolicited manner; that is, clinicians included this topic in the conversation not in response to questions from parents.

Many clinicians expressed strong support for HPV vaccination while at the same time describing how they qualified their recommendation with statements about it not being required for school. Clinicians often noted that HPV vaccination was important for providing excellent protection against cancers and that they supported HPV vaccination for staying healthy, yet also mentioned that it was an additional vaccine that was not required.

A few clinicians felt that the lack of a requirement resulted in the need for longer conversations with parents to obtain their acceptance. Some clinicians noted that the lack of a requirement resulted in parents taking more time to consent.

Clinicians expressed varied opinions about how their recommendations for HPV vaccination would be different if school entry requirements were enacted. Many clinicians felt that requirements would shorten and simplify the recommendation. They recognized that there was usually less discussion and fewer questions about required immunizations, and that requirements made it easier for some parents to accept vaccination. This sentiment was also expressed by clinicians across the range of practice settings. Notably, some clinicians acknowledged that conversation about the vaccine would still be important.

Other clinicians described how the conversation could become more difficult. Recognizing that HPV is not transmitted through casual, non-intimate contact, some clinicians felt that they might experience resistance from parents who would not agree with a requirement for school entry. Specifically, it was noted that parents might be annoyed by a requirement for vaccination against a sexually transmitted infection. Relatively more clinicians from private practices felt that the conversation would be more difficult and/or lengthy due to push back from parents who might be offended by the requirement.

Few clinicians explained how their recommendations would not change if there were a school requirement. These clinicians remarked that all vaccines were treated the same, and that the rationale for all vaccines should be explained.

The majority of clinicians in all practice settings believed that more parents would accept vaccination if it were required for school entry. Some clinicians felt that this would directly remove the sense of vaccination being optional. Other clinicians felt that some parents would be happy to follow their clinicians' recommendations for a required vaccine without too much extra consideration. Clinicians also felt that the recommendations would signal the importance of HPV vaccination to parents. In response to the general question about what would help to increase coverage, some clinicians responded that school entry requirements were the most obvious way to achieve this goal.

Clinicians did discuss how a school requirement could have different degrees of impact on coverage. Some felt that it would help a relatively small number of patients, or just the more reluctant families. Others felt that acceptance would go up significantly and that it could help to vaccinate a large proportion of the population. Even in the context of anticipated parental pushback, several clinicians described how a school requirement would still increase coverage. Only one clinician expressed the dissenting opinion that it would not make a substantial difference.

Clinicians' own personal opinions about HPV vaccination school entry requirements ranged the spectrum from positive to negative. Some clinicians in both public and private settings described being unequivocally in favour of requirements. Others expressed their own personal support for a requirement but with accompanying concerns about whether society was ready for such a requirement, or whether it would be appropriate for everyone. Some clinicians were more ambivalent in their attitudes about a school requirement for HPV vaccine. A smaller number of clinicians, mostly in private practice, were not in favour of school requirements. The main reason was concern about loss of parental autonomy in making health care decisions.

\section{Discussion}

Results of this study indicate that the current lack of middle school entry requirements is a powerful influence on clinicians' recommendations for HPV vaccination. This is significant because a recommendation from a clinician is one of the most salient determinants of HPV vaccine uptake. ${ }^{9}, 10$ A high quality recommendations for HPV vaccination has been described as one that is strong (emphasizing the importance of vaccination), urgent (recommending same-day vaccination), consistent (recommending vaccination routinely), and timely (recommending vaccination by ages 11-12 years). ${ }^{15}$ Discussions about not needing HPV vaccination to attend school may make this vaccine seem non-urgent, and thus could be viewed as being of lower quality. Interestingly, many clinicians provide strong recommendations, another component of a high-

Table 4. Personal opinions.

\begin{tabular}{ll} 
Themes & Illustrative quotes \\
In favour & It would absolutely be a positive change. (P23) \\
& I would love that. (P26) \\
Mixed/ambivalent feelings & That [requirements] would be great, but mandates are tough...I don't think we've got \\
& our ducks in a - I wasn't necessarily embracing doing it now. I don't think we've got our ducks in a row \\
& to do it. I'd hate to see it - I don't want to see that effort fail if I didn't really think we were ready. (P4) \\
& I think parents may accept it more, may get more - more people - \\
& but I'm not sure if I agree that it should be mandated. (P18) \\
& Am I for it? In the general population, I'd say yes. But there would be exceptions. (P22) \\
& I could see both sides. (P24) \\
& It's just doing things against their beliefs. I think a parent has the right to decide their - \\
& to make their own decisions about the vaccine. (P6) \\
& The less the government interferes with health care, the better. (P25) \\
\hline Not in favour &
\end{tabular}


quality recommendation, by emphasizing its importance for preventing cancer, while simultaneously conveying a lack of urgency. Thus, in the current absence of requirements for HPV vaccination for school entry in most places (48 out of 51 jurisdictions), renewed efforts are needed to strengthen the quality of clinicians' recommendations in a way that is strongly focused on cancer prevention and uncoupled from lack of requirements that may encourage delays.

Several of the key findings from this study were expressed across the range of practice types from public to private. For example, statements were widespread that recommendations for HPV vaccine were different from other vaccines due to lack of requirements, and that school requirements would increase coverage. On other topics, however, differences emerged. The possibility that longer conversations might be needed with parents who have concerns about school requirements were largely raised by clinicians who care mostly for privately insured patients. This may reflect higher levels of vaccine hesitancy among this population. ${ }^{26}$ Clinicians in these practice environments may also feel greater fiscal pressures for more efficient visits from insurers and administrators. ${ }^{27}$ These time pressures could be an important force in clinicians' reluctance to engage in potentially lengthy conversations about the need for HPV vaccination, and it could be further compounded by different perceptions about the need for vaccination based on suspected sexual activity. ${ }^{16}$ Efforts to work with clinicians to strengthen recommendations may need to address unique situations in different practice settings.

Reasons for the widespread lack of requirements for HPV vaccination are complex and likely include the persistent reluctance of states to revisit early efforts at enacting requirements that failed for predominantly political reasons. ${ }^{28,29}$ If states consider enacting requirements as a next step for increasing both coverage and equity, it will be important for legislators and policy makers to engage with key stakeholder groups including clinicians who have the primary responsibility for immunizing children and adolescents, as they will play an important role in this effort, both through their support of enacting legislation and their subsequent efforts to increase compliance with the new regulations. ${ }^{23}$ The mixed personal opinions and some degree of ambivalence among clinicians about HPV vaccination requirements that were observed in this study and previously reported by others indicate that efforts are needed to build support for such a policy change to be successful. ${ }^{24,30-32}$ Clearly, more than 10 years after vaccine introduction, this hesitancy lingers despite the fact that requirements for other adolescent vaccines were enacted well within 10 years in a majority of jurisdictions. ${ }^{29}$

A key reason cited by clinicians in this study for not supporting school entry requirements was related to valuing individual autonomy in decision making over government interference. In the case of HPV vaccine, this sentiment may be even stronger because of the perceived lack of risk of transmission in the school setting. Activities to promote greater acceptance might include broad educational efforts targeted toward clinicians about how requirements for other vaccines have greatly increased coverage and reduced disparities. ${ }^{19,20,33}$ The recent experience in Rhode Island may signal the potential for requirements to increase coverage for HPV vaccination specifically. In 2015, Rhode Island became the third jurisdiction to pass a school entry requirement for HPV vaccination after Virginia and the District of Columbia enacted requirements in 2008 and 2009, respectively. ${ }^{21}$ After school entry requirements took effect, some public backlash occurred, but coverage then increased in the following year from $76 \%$ to $90 \%$ in adolescent girls and $69 \%$ to $88 \%$ in adolescent boys for at least 1 dose. ${ }^{34}$

\section{Limitations}

A key limitation of this study is the potential lack of generalizability. First, all clinicians were recruited from a single state and there could be variability in views and opinions across other regions of the US. Second, our sampling strategy did not capture the full range of clinicians who provide immunizations (for example, physician assistants), thus our results may not reveal the complete landscape of views. Local data may be helpful in other areas to fully inform clinician interventions and policy approaches going forward.

\section{Conclusions}

The current lack of school entry requirements for HPV vaccination is an important influence on clinicians' recommendations that are often framed as optional or non-urgent. Efforts are needed to strengthen the quality of clinicians' current recommendations in a way that is focused on prevention of cancer, inarguably an important individual and public health goal, and uncoupled from the lack of school entry requirements that may create a sense of non-urgency. Additionally, greater support for requirements among clinicians may be needed to successfully enact school entry requirements in the future. This may be a challenge for a vaccine that has raised unique political and social debates, but likely a worthy goal to protect adolescents from future cancers.

Correspondence: Linda M. Niccolai, Yale School of Public Health, 60 College Street, New Haven CT, 06520 USA.

Tel.: +1.203.785.7834 - Fax: +1.203 .785 .3604$

E-mail: linda.niccolai@yale.edu

Key words: human papillomavirus vaccination, clinicians, school entry requirements.

Contributions: LMN, ALN, CEH, conception of study; ALN, CEH, data collection; LMN, drafting the article; LMN, ALN, AF, CEH data analysis and interpretation, critical revision of article, final approval to be submitted for publication.

Conflict of interest: the authors declare no potential conflict of interest. Funding: this work was supported by a grant from National Cancer Institute at US National Institutes of Health (P30CA016359-35S4). Funders played no role in the design, conduct, analysis or interpretation of this study, or the decision to submit for publication.

Received for publication: 28 November 2017.

Revision received: 23 February 2018.

Accepted for publication: 5 March 2018.

CC Copyright L.M. Niccolai et al., 2018

Licensee PAGEPress, Italy

Journal of Public Health Research 2018;7:1324

doi:10.4081/jphr.2018.1324

This work is licensed under a Creative Commons Attribution NonCommercial 4.0 License (CC BY-NC 4.0).

\section{References}

1. Centers for Disease Control and Prevention. Human papillomavirus-associated cancers - United States, 2004 - 2008. MMWR 2012;61:258-61.

2. Schiller JT, Castellsague X, Garland SM. A review of clinical trials of human papillomavirus vaccines. Vaccine 2012;30S:F123-F138.

3. Drolet M, Benard E, Boily M-C, et al. Population-level impact 
and herd effects following human papillomavirus vaccination programmes: a systematic review and meta-analysis. Lancet Infect Dis 2015;15:565-80.

4. Centers for Disease Control and Prevention. National, Regional, State, and Selected Local Area Vaccination Coverage Among Adolescents Aged 13-17 Years - United States, 2016. MMWR 2017;66:874-82.

5. Bruni L, Diaz M, Barrionuevo-Rosas L, et al. Global estimates of human papillomavirus vaccination coverage by region and income level: a pooled analysis. Lancet Glob Health 2016;4:e453-63.

6. Shapiro GK, Guichon J, Kelaher M. Canadian school-based HPV vaccine programs and policy considerations. Vaccine 2017;35:5700-7.

7. Jean S, Elshafei M, Buttenheim A. Social determinants of community-level human papillomavirus vaccination coverage in a school-based vaccination program. Sex Transm Infect 2018.

8. Brotherton JM, Murray SL, Hall MA, et al. Human papillomavirus vaccine coverage among female Australian adolescents: success of the school-based approach. Med J Aust 2013;199:614-7.

9. Holman DM, Benard V, Roland KB, et al. Barriers to human papillomavirus vaccination among US adolescents: a systematic review of the literature. JAMA Pediatr 2014;168:76-82.

10. Rambout L, Tashkandi M, Hopkins L, Tricco AC. Self-reported barriers and facilitators to preventive human papillomavirus vaccination among adolescent girls and young women: a systematic review. Prev Med 2014;58:22-32.

11. Niccolai LM, Hansen CE, Credle M, Shapiro ED. Parents' recall and reflections on experiences related to HPV vaccination for their children. Qual Health Res 2016;26:842-50.

12. Henrikson NB, Tuzzio L, Gilkey MB, McRee AL. You're never really off time: Healthcare providers' interpretations of optimal timing for HPV vaccination. Prev Med Rep 2016;4:94-7.

13. Gilkey MB, Calo WA, Moss JL, et al. Provider communication and HPV vaccination: The impact of recommendation quality. Vaccine 2016;34:1187-92.

14. Gilkey MB, Moss JL, Coyne-Beasley T, et al. Physician communication about adolescent vaccination: How is human papillomavirus vaccine different? Prev Med 2015;77:181-5.

15. Gilkey MB, Malo TL, Shah PD, et al. Quality of physician communication about human papillomavirus vaccine: findings from a national survey. Cancer Epidemiol Biomark Prev 2015;24:1673-9.

16. Perkins RB, Clark JA, Apte G, et al. Missed opportunities for HPV vaccination in adolescent girls: a qualitative study. Pediatrics 2014;134:e666-74.

17. Niccolai LM, Pettigrew M. The role of cognitive bias in suboptimal HPV vaccine uptake. Pediatrics 2016;138:e20161537.

18. Healy CM, Montesinos DP, Middleman AB. Parent and provider perspectives on immunization: are providers overestimating parental concerns? Vaccine 2014;32:579-84.

19. Orenstein WA, Hinman AR. The immunization system in the
United States - the role of school immunization laws. Vaccine 1999; 17:S19-24.

20. Morita JY, Ramirez E, Trick WE. Effect of a school-entry vaccination requirement on racial and ethnic disparities in hepatitis B immunization coverage levels among public school students. Pediatrics. 2008;121:e547-52.

21. Immunization Action Coalition. State information: HPV mandates for children in secondary schools. Available from: http://www.immunize.org/laws/hpv.asp. Accessed 10/12/2017.

22. Schwartz JL, Caplan AL, Faden RR, Sugarman J. Lessons from the failure of human papillomavirus vaccine state requirements. Clin Pharm Therap 2007;82:760-3.

23. North AL, Niccolai LM. Human papillomavirus vaccination requirements in US schools: recommendations for moving forward. Am J Public Health 2016.303286.

24. Vercruysse J, Chigurupati NL, Fung L, et al. Parents' and providers'attitudes toward school-located provision and school-entry requirements for HPV vaccines. Human Vacc Immun 2016;1140289.

25. Guest G, MacQueen KM, Namey EE. Applied Thematic Analysis. SAGE Publications, Inc, Thousand Oaks CA. 2014.

26. Hough-Telford C, Kimberlin DW, Aban I, et al. Vaccine delays, refusals, and patient dismissals: a survey of pediatricians. Pediatrics 2016;138:e20162127.

27. Halfon N, Stevens GD, Larson K, Olson LM. Duration of a well-child visit: association with content, family-centeredness, and satisfaction. Pediatrics 2011;128;657-64.

28. National Council of State Legislatures. HPV vaccine: legislation and statues. http://www.ncsl.org/research/health/hpv-vaccine-state-legislation-and-statutes.aspx. Accessed November 4, 2016.

29. Schwartz JL, Easterling LA. State vaccination requirements for HPV and other vaccines for adolescents, 1990-2015. JAMA. 2015;314:1-2.

30. Califano S, Calo WA, Weinberger M, et al. Physician support of HPV vaccination school-entry requirements. Human Vacc Immun 2016;12:1626-32.

31. Perkins RB, Clark JA. What affects human papillomavirus vaccination rates? A qualitative analysis of providers' perceptions. Women Health Issue 2012;22-4; 379-86.

32. Kahn JA, Cooper HP, Vadaparampil ST, et al. Human papillomavirus vaccine recommendations and agreement with mandated human papillomavirus vaccination for 11-to-12 year old girls: a statewide survey of Texas physicians. Cancer Epidemiol Biomarkers Prev 2009;18:2325-32.

33. Kharbanda EO, Stockwell MS, Colgrove J, et al. Changes in Tdap and MCV4 vaccine coverage following enactment of a statewide requirement of Tdap vaccination for entry into sixth grade. Am J Public Health 2010;100:1635-40.

34. Centers for Disease Control and Prevention. National, Regional, State, and Selected Local Area Vaccination Coverage Among Adolescents Aged 13-17 Years - United States, 2014. MMWR 2015;64:784-92. 